\title{
A phase II study of FOLFOXIRI plus bevacizumab as initial chemotherapy for patients with untreated metastatic colorectal cancer: TRICC1414 (BeTRI)
}

\author{
Katsunori Shinozaki ${ }^{1}\left[\right.$. Takeshi Yamada ${ }^{2}$ Junichiro Nasu $^{3} \cdot$ Toshihiko Matsumoto $^{4}$. Yasuhiro Yuasa ${ }^{5}$. \\ Takeshi Shiraishi $^{6} \cdot$ Hiroaki Nagano $^{7} \cdot$ Ichiro Moriyama ${ }^{8} \cdot$ Toshiyoshi Fujiwara $^{9} \cdot$ Masashi Miguchi $^{10}$. \\ Ryosuke Yoshida ${ }^{11} \cdot$ Kimiyasu Nozaka $^{12}$. Hiroaki Tanioka ${ }^{13}$. Takeshi Nagasaka ${ }^{13} \cdot$ Yasuro Kurisu $^{14}$. \\ Michiya Kobayashi ${ }^{15} \cdot$ Kenji Tsuchihashi $^{16} \cdot$ Michio Inukai $^{3} \cdot$ Takashi Kikuchi $^{17} \cdot$ Tomohiro Nishina $^{18}$
}

Received: 16 August 2020 / Accepted: 9 October 2020 / Published online: 23 October 2020

(c) The Author(s) 2020

\begin{abstract}
Purpose FOLFOXIRI plus bevacizumab is regarded as a first-line therapeutic option for selected patients with metastatic colorectal cancer (mCRC). Our aim was to assess the efficacy and safety of induction treatment with FOLFOXIRI plus bevacizumab in patients with untreated mCRC harboring $U G T 1 A 1$ wild $(* 1 / * 1)$, or single-hetero $(* 1 / * 6$ or $* 1 / * 28)$ genotypes. Methods Twelve cycles of FOLFOXIRI plus bevacizumab were administered to patients with untreated mCRC. The primary endpoint was the overall response rate (ORR) assessed by central independent reviewers. Secondary endpoints included time to treatment failure (TTF), progression-free survival (PFS), overall survival (OS), relative dose intensity (RDI), R0 resection rate, and safety. The exploratory objectives were early tumor shrinkage (ETS) and depth of response (DoR).

Results Of the 47 patients enrolled, 46 and 44 patients were eligible for the safety and efficacy analysis, respectively. The primary endpoint was met. The ORR was $63.6 \%$ (95\% CI 47.8-77.6). At a median follow-up of 25.4 months, median TTF, PFS, and OS was 8.1, 15.5, and 34.4 months, respectively. The median RDI of 5-fluorouracil, irinotecan, oxaliplatin, and bevacizumab was $72,69,62$, and $71 \%$, respectively. R0 resection rate was $22.7 \%$. Grade 3 or higher adverse events $(\geq 10 \%)$ included neutropenia (65.2\%), febrile neutropenia (26.1\%), leukopenia (23.9\%), anorexia (10.9\%), nausea (10.9\%), and diarrhoea (10.9\%). No treatment-related deaths were observed. ETS and DoR were 70.5 and $45.4 \%$, respectively.

Conclusions FOLFOXIRI plus bevacizumab induction treatment of Japanese patients was shown to be beneficial and manageable, although caution is required since the treatment causes febrile neutropenia.
\end{abstract}

Keywords FOLFOXIRI plus bevacizumab $\cdot$ Metastatic colorectal cancer $\cdot$ Efficacy $\cdot$ Conversion surgery $\cdot$ Safety

\section{Introduction}

Colorectal cancer (CRC) is the third most common malignant neoplasm and the second major cause of cancer death in 2018 worldwide for both sexes [1]. Most patients with metastatic CRC (mCRC) have unresectable disease and cannot be cured. However, long-term survival or even cure is

Electronic supplementary material The online version of this article (https://doi.org/10.1007/s10147-020-01811-w) contains supplementary material, which is available to authorized users.

Katsunori Shinozaki

k-shinozaki@hph.pref.hiroshima.jp

Extended author information available on the last page of the article reported to have been attained in $20-50 \%$ of patients who underwent complete R0 resection of their metastases [2].

The Gruppo Oncologico Nord Ovest (GONO) group's phase III TRIBE trial compared FOLFOXIRI plus bevacizumab versus FOLFIRI plus bevacizumab in unresectable mCRC patients in Italy [3]. This trial demonstrated that FOLFOXIRI plus bevacizumab increased ORR (65 vs. $53 \%, p=0.006)$ and PFS (12.1 vs. 9.7 months, $p=0.003)$. An updated analysis on the TRIBE trial reported that OS was prolonged (29.8 vs. 25.8 months, $p=0.03$ ) [4].

The major clinical practice guidelines, including the Japanese Society for Cancer of the Colon and Rectum (JSCCR) guideline 2019, all recommend FOLFOXIRI plus bevacizumab as a first-line therapeutic option for selected patients with mCRC [5-8]. Only two prospective, single-arm phase 
II trials of FOLFOXIRI plus bevacizumab have been conducted to assess the safety and efficacy in Japan. One is the QUATTRO study using the GONO-FOLFOXIRI regimen [9]. The other is the JACCRO-CC11 trial using a modified FOLFOXIRI regimen that consisted of oxaliplatin $(85 \mathrm{mg} /$ $\left.\mathrm{m}^{2}\right)$, irinotecan $\left(150 \mathrm{mg} / \mathrm{m}^{2}\right)$, l-leucovorin $\left(200 \mathrm{mg} / \mathrm{m}^{2}\right)$, and 5-fluorouracil $\left(2400 \mathrm{mg} / \mathrm{m}^{2}\right)$ [10]. Both studies concluded that FOLFOXIRI plus bevacizumab was effective. The incidence of febrile neutropenia was as low as $5 \%$ in modified FOLFOXIRI compared to $21.7 \%$ in GONO-FOLFOXIRI. However, mature PFS and OS have not been documented for either of these studies. The recommended doses of FOLFOXIRI also remain controversial.

Adverse events associated with the FOLFOXIRI regimen include higher risks of neutropenia and diarrhoea [11]. Irinotecan especially has significant adverse effects, including myelosuppression and diarrhoea. In Japanese patients, homozygosity for $U G T 1 A 1 * 28$ or $U G T 1 A 1 * 6$ and heterozygosity for both $U G T 1 A 1 * 6$ and $U G T 1 A 1 * 28$ are associated with severe irinotecan-related neutropenia $[12,13]$.

Thus, we conducted a single-arm, multicenter, phase II study to assess the efficacy and safety of induction treatment with FOLFOXIRI plus bevacizumab in patients with untreated mCRC harboring UGT1A1 wild $(* 1 / * 1)$ or singlehetero $(* 1 / * 6$ or $* 1 / * 28)$ genotypes. In our study, sequential treatment with the remaining drugs was continued after termination of protocol therapy or discontinuation of oxaliplatin and/or irinotecan at the investigator's discretion.

\section{Patients and methods}

\section{Study design}

The Bevacizumab plus Triplet (BeTRI) study was a multisite, open-label, single-arm, phase II clinical trial. This trial was registered on the UMIN Clinical Trials Registry (UMIN000017102), followed by Japan Registry of Clinical Trials (jRCTs061180021).

\section{Patients}

Patients who met the following criteria were enrolled in this study: aged between 20 and 70 years; histologically confirmed adenocarcinoma of the colon or rectum; unresectable or recurrent CRC patient; no prior chemotherapy, immunotherapy, or radiation therapy (but can be enrolled 6 months after the date of completion of adjuvant chemotherapy); one or more measurable lesions; ECOG PS of 0 or 1; patients harbored $U G T 1 A 1$ wild $\left(* 1 /{ }^{*} 1\right)$ or single-heterozygous
$(* 1 / * 6$ or $* 1 / * 28)$ genotypes. Patients were ineligible if they had severe, uncontrolled organ or metabolic dysfunction.

\section{Endpoints}

The primary endpoint was the objective response rate (ORR) assessed by central independent reviewers. Secondary endpoints were TTF and PFS, OS, R0 resection rate, relative dose intensity (RDI), and safety. TTF represented the time from the initial day of protocol therapy to the first day when we observed any of the following events: discontinuation of protocol therapy; initial progression; death with any cause. PFS was defined as the time from the initial day of protocol therapy to the initial progression day or death for any reason. The exploratory objectives were early tumor shrinkage (ETS) and depth of response (DoR).

\section{Treatment and evaluation}

We defined protocol therapy as 12 cycles of FOLFOXIRI plus bevacizumab induction treatment consisting of a 30-90-min infusion of bevacizumab at a dose of $5 \mathrm{mg} / \mathrm{kg}$, a 90 -min infusion of irinotecan at a dose of $165 \mathrm{mg} / \mathrm{m}^{2}$, a 120 -min infusion of oxaliplatin at a dose of $85 \mathrm{mg} / \mathrm{m}^{2}$ and a concomitant 120 -min infusion of $l$-leucovorin at a dose of $200 \mathrm{mg} / \mathrm{m}^{2}$. These were followed by a $48-\mathrm{h}$ continuous infusion of 5-fluorouracil to a total dose of $3200 \mathrm{mg} / \mathrm{m}^{2}$. Cycles were repeated every 14 days.

The chemotherapy was continued until disease progression, an unacceptable adverse event, tumor resection, a delay of more than 29 days due to an adverse event, reduction of 5-fluorouracil dose to less than $2000 \mathrm{mg} / \mathrm{m}^{2}$, or consent withdrawal. In cases of prespecified adverse events, treatment modification was permitted according to the study protocols (Supplementary Tables 1 and 2). If oxaliplatin and/or irinotecan was discontinued at the investigator's discretion, sequential treatment consisting of the remaining drugs was continued.

We analyzed safety with a safety analysis set (SAS) in which patients received at least one cycle of protocol therapy. Efficacy was analyzed with a full analysis set (FAS) consisting of eligible patients. Based on the Response Evaluation Criteria in Solid Tumors (RECIST) version 1.1, central independent reviewers assessed response and progression, referring to CT or MRI taken every 8 weeks. ETS was defined as a reduction of at least $20 \%$ in the sum of the longest diameters of target lesions at week 8 compared with baseline. DoR was defined as the relative change in the sum of the longest diameters of RECIST target lesions at the nadir, in the absence of new lesions or progression of non-target lesions, when compared with 
baseline. Adverse events were evaluated according to CTCAE version 4.0.

\section{Sample size}

We assumed the expected response rate to be $70 \%$ and the threshold response rate to be $45 \%$, with a two-sided $\alpha$ error of 0.05 and a power of $90 \%$. The expected response rate was grounded in the results of the GONO and TRIBE trials, of which the confirmed ORRs were 65 and $77 \%$ for FOLFOXIRI plus bevacizumab, respectively [3, 14]. We also referred to the threshold response rate reported by the NO16966 and TRIBE trials, of which the confirmed ORRs were 34 and $53 \%$ for doublet plus bevacizumab, respectively [3, 15]. We estimated a minimum of 40 patients, but we planned to enrol 45 or more patients, taking account of dropouts and withdrawals.

\section{Statistical analyses}

We concluded that the treatment could be regarded as promising if the lower limit of the $95 \% \mathrm{CI}$ exceeded $45 \%$. For the evaluation of the ORR and ETS, we calculated the $95 \%$ CI of the rate using the Clopper-Pearson method with F distribution. We used the Kaplan-Meier method to estimate TTF, PFS, and OS. Statistical analyses were carried out using SAS version 9.4 (SAS Institute Inc., Cary, NC, USA) and R $\times 64$ version 3.5.2.

\section{Results}

\section{Patients}

Between April 2015 and May 2017, a total of 47 patients were enrolled from 16 Japanese study sites. One patient was excluded from the study because of a deviation of the inclusion criteria. Two more patients were removed after the central reviewers determined there were no measurable lesions. The numbers in the SAS and FAS were, therefore, 46 and 44, respectively (Supplementary Fig. 1).

Table 1 shows patient characteristics in the SAS. There were twenty-six male and twenty female patients with a median age of 58 years (range 29-68 years). ECOG PS scores of 0 and 1 were $76 \%$ and $24 \%$, respectively. The site of the primary tumor was right colon in $10(22 \%)$ patients, left colon in $16(35 \%)$, and rectum in $20(43 \%)$ patients. Resection rate of the primary tumor was $37 \%$. Seventeen (37\%), 24 (52\%) and $5(11 \%)$ patients had $R A S$ wild-type, $R A S$ mutated, and $R A S$ unknown tumor, respectively.
Table 1 Baseline characteristics

\begin{tabular}{|c|c|c|}
\hline & Patients $(n=46)$ & $\%$ \\
\hline Age (years; median, range) & $58(29-68)$ & \\
\hline \multicolumn{3}{|l|}{ Sex } \\
\hline Male & 26 & 57 \\
\hline Female & 20 & 43 \\
\hline \multicolumn{3}{|l|}{ ECOG PS } \\
\hline 0 & 35 & 76 \\
\hline 1 & 11 & 24 \\
\hline \multicolumn{3}{|l|}{ Site of primary tumor } \\
\hline Right colon & 10 & 22 \\
\hline Left colon & 16 & 35 \\
\hline Rectum & 20 & 43 \\
\hline \multicolumn{3}{|l|}{ Resection of primary tumor } \\
\hline Yes & 17 & 37 \\
\hline No & 29 & 63 \\
\hline \multicolumn{3}{|l|}{ Site of metastases* } \\
\hline Liver & 36 & 78 \\
\hline Lung & 13 & 28 \\
\hline Lymph node & 7 & 15 \\
\hline Peritoneum & 4 & 9 \\
\hline \multicolumn{3}{|l|}{$U G T 1 A 1$} \\
\hline Wild type $(* 1 / * 1)$ & 24 & 52 \\
\hline Single heterozygous $(* 1 / * 6$ or $* 1 / * 28)$ & 22 & 48 \\
\hline \multicolumn{3}{|l|}{$R A S$} \\
\hline Wild type & 17 & 37 \\
\hline Mutated & 24 & 52 \\
\hline Unknown & 5 & 11 \\
\hline
\end{tabular}

ECOG PS Eastern Cooperative Oncology Group performance status, Right colon cecum, ascending and transverse colon, Left colon descending and sigmoid colon

*Site of metastases were counted in duplicate

\section{Study treatment}

All 46 enrolled patients received FOLFOXIRI plus bevacizumab. The commencement of second cycle treatment was delayed in $33(72 \%)$ patients. In addition, the dose level was reduced in the second cycle in $23(50 \%)$ patients. Meanwhile, twelve (26\%) patients completed the second cycle treatment as scheduled without dose modification.

A total of 27 (59\%) patients completed twelve cycles of protocol therapy. Table 2 shows the details of the treatment after protocol therapy. At the twelfth cycle, 18 (39.1\%) patients received FOLFOXIRI plus bevacizumab, six (13.0\%) patients received FOLFIRI plus bevacizumab, two (4.3\%) patients received FOLFIRI, and one (2.2\%) patient received infusional 5-fluorouracil and leucovorin plus bevacizumab. The protocol therapy was discontinued in 9 (20\%) patients due to adverse events, in $8(17 \%)$ patients due to surgery, in one (2\%) patient due to progressive 
Table 2 Reasons of protocol discontinuation and post-protocol treatment details

\begin{tabular}{llllr}
\hline $\begin{array}{l}\text { Reason of protocol } \\
\text { discontinuation }\end{array}$ & $n=46$ & Post-treatment details & $n=46$ & $\%$ \\
\hline Surgery & 8 & R0 resection & 5 & 10.9 \\
& & R2 resection & 3 & 6.5 \\
Patient's withdrawal & 1 & FOLFIRI + Bmab & 1 & 2.2 \\
Disease progression & 1 & FOLFOXIRI+Bmab & 1 & 2.2 \\
Adverse event & 9 & FOLFOXIRI \pm Bmab & 4 & 8.7 \\
& & FOLFIRI+Bmab & 2 & 4.3 \\
& & FL+Bmab & 1 & 2.2 \\
& & Capecitabine + Bmab & 1 & 2.2 \\
Protocol completion* & 27 & BSC & 1 & 2.2 \\
& & FOLFOXIRI + Bmab & 18 & 39.1 \\
& & FOLFIRI \pm Bmab & 8 & 17.4 \\
& & FL + Bmab & 1 & 2.2 \\
\hline
\end{tabular}

*Treatment performed in the 12th cycle of protocol therapy

RO resection curative resection, Bmab bevacizumab, $F L$ infusional 5-fluorouracil and leucovorin, $B S C$ best supportive care

disease, and in one (2\%) patient due to withdrawal of consent. Of the nine patients for whom protocol therapy was discontinued due to adverse events, eight patients resumed chemotherapy, and their treatments (number of patients) were as follows: FOLFOXIRI plus bevacizumab (3); FOLFOXIRI (1); FOLFIRI plus bevacizumab (2); Capecitabine plus bevacizumab (1); and 5-fluorouracil and leucovorin plus bevacizumab (1).

Although second-line treatments were not specified by the protocol, we requested that treatment administered after progression of protocol therapy and treatment with drugs not included in the protocol therapy (i.e. second-line treatments) must be reported. Second-line chemotherapy (cases per 16 patients total) were as follows: FOLFIRI plus anti-VEGF/ VEGFR (4); FOLFOXIRI plus or minus bevacizumab (3); anti-EGFR plus or minus irinotecan (3); capecitabine plus or minus bevacizumab (3); irinotecan plus tegafur/gimeracil/oteracil (1); regorafenib (1); and trifluridine/tipiracil (1) (Supplementary Table 4).

In the FAS, the median relative dose intensity of 5-fluorouracil, irinotecan, oxaliplatin, and bevacizumab was 72 , 69,62 and $71 \%$, respectively. The median number of cycles administered per patient as FOLFOXIRI plus or minus bevacizumab was eight (range 2-12).

\section{Efficacy}

The efficacy results are summarized in Table 3. Because CR and PR were achieved in $1(2.3 \%)$ patient and 27 (61.4\%) patients, respectively, the ORR was $63.6 \%$ (95\% CI 47.8-77.6). The numbers (\%) of patients with stable disease (SD) and with progressive disease (PD) were 14 (31.8\%) and $2(4.5 \%)$, respectively. In addition, the ORR at week 8
Table 3 Tumor response (ORR, ETS, DoR), TTF, PFS, and OS

\begin{tabular}{|c|c|c|c|c|c|}
\hline \multirow[t]{2}{*}{ Variable } & \multirow{2}{*}{$\begin{array}{l}\text { All } \\
(n=44)\end{array}$} & \multicolumn{2}{|l|}{ Location } & \multicolumn{2}{|l|}{$R A S$ status* } \\
\hline & & $\begin{array}{l}\text { Right-sided colon } \\
(n=10)\end{array}$ & $\begin{array}{l}\text { Left-sided colon } \\
(n=34)\end{array}$ & $\begin{array}{l}\text { Wild } \\
(n=15)\end{array}$ & $\begin{array}{l}\text { Mutated } \\
(n=24)\end{array}$ \\
\hline \multicolumn{6}{|c|}{ Overall response rate (ORR) } \\
\hline $\begin{array}{l}\text { No. of pts }(\%) \\
(95 \% \text { CI })\end{array}$ & $\begin{array}{l}28(63.6) \\
(47.8-77.6)\end{array}$ & $\begin{array}{l}7(70.0) \\
(34.8-93.3)\end{array}$ & $\begin{array}{l}21(61.8) \\
(43.6-77.8)\end{array}$ & $\begin{array}{l}11(73.3) \\
(44.9-92.2)\end{array}$ & $\begin{array}{l}15(62.5) \\
(40.6-81.2)\end{array}$ \\
\hline \multicolumn{6}{|c|}{ Early tumor shrinkage (ETS) } \\
\hline $\begin{array}{l}\text { No. of pts (\%) } \\
(95 \% \mathrm{CI})\end{array}$ & $\begin{array}{l}31(70.5) \\
(54.8-83.2)\end{array}$ & $\begin{array}{l}7(70.0) \\
(34.8-93.3)\end{array}$ & $\begin{array}{l}24(70.6) \\
(52.5-84.9)\end{array}$ & $\begin{array}{l}11(73.3) \\
(44.9-92.2)\end{array}$ & $\begin{array}{l}17(70.8) \\
(48.9-87.4)\end{array}$ \\
\hline \multicolumn{6}{|c|}{ Deepness of response (DoR) } \\
\hline $\begin{array}{l}\text { Median \% } \\
(95 \% \mathrm{CI})\end{array}$ & $\begin{array}{l}45.4 \\
(37.8-49.9)\end{array}$ & $\begin{array}{l}44.7 \\
(31.1-64.7)\end{array}$ & $\begin{array}{l}45.4 \\
(36.1-49.3)\end{array}$ & $\begin{array}{l}47.6 \\
(39.3-61.1)\end{array}$ & $\begin{array}{l}45.2 \\
(34.1-50.8)\end{array}$ \\
\hline \multicolumn{6}{|c|}{ Time to treatment failure (TTF) } \\
\hline $\begin{array}{l}\text { Median months } \\
(95 \% \mathrm{CI})\end{array}$ & $\begin{array}{l}8.1 \\
(5.3-10.1)\end{array}$ & $\begin{array}{l}11.5 \\
(8.2-24.1)\end{array}$ & $\begin{array}{l}6.4 \\
(4.2-9.7)\end{array}$ & $\begin{array}{l}11.4 \\
(7.6-15.5)\end{array}$ & $\begin{array}{l}6.8 \\
(4.2-9.3)\end{array}$ \\
\hline \multicolumn{6}{|c|}{ Progression-free survival (PFS) } \\
\hline $\begin{array}{l}\text { Median months } \\
(95 \% \mathrm{CI})\end{array}$ & $\begin{array}{l}15.5 \\
(11.5-23.4)\end{array}$ & $\begin{array}{l}24.1 \\
(11.5-26.2)\end{array}$ & $\begin{array}{l}14.9 \\
(10.1-22.9)\end{array}$ & $\begin{array}{l}13.2 \\
(11.4-24.1)\end{array}$ & $\begin{array}{l}17.9 \\
(13.7-26.2)\end{array}$ \\
\hline \multicolumn{6}{|c|}{ Overall survival (OS) } \\
\hline $\begin{array}{l}\text { Median months } \\
(95 \% \text { CI })\end{array}$ & $\begin{array}{l}34.4 \\
(26.4-\mathrm{NR})\end{array}$ & $\begin{array}{l}26.4 \\
(26.2-\mathrm{NR})\end{array}$ & $\begin{array}{l}34.4 \\
(23.6-\mathrm{NR})\end{array}$ & $\begin{array}{l}37.1 \\
(23.6-\mathrm{NR})\end{array}$ & $\begin{array}{l}29.8 \\
(26.2-\mathrm{NR})\end{array}$ \\
\hline
\end{tabular}

Right-sided colon cecum, ascending and transverse colon, Left-sided colon descending, sigmoid colon, and rectum, No. number, $p t s$ patients, NR not reached

*Five patients with unknown $R A S$ status were excluded 
Fig. 1 Waterfall plot showing maximum percentage change from baseline in size of tumors assessed by central independent reviewers

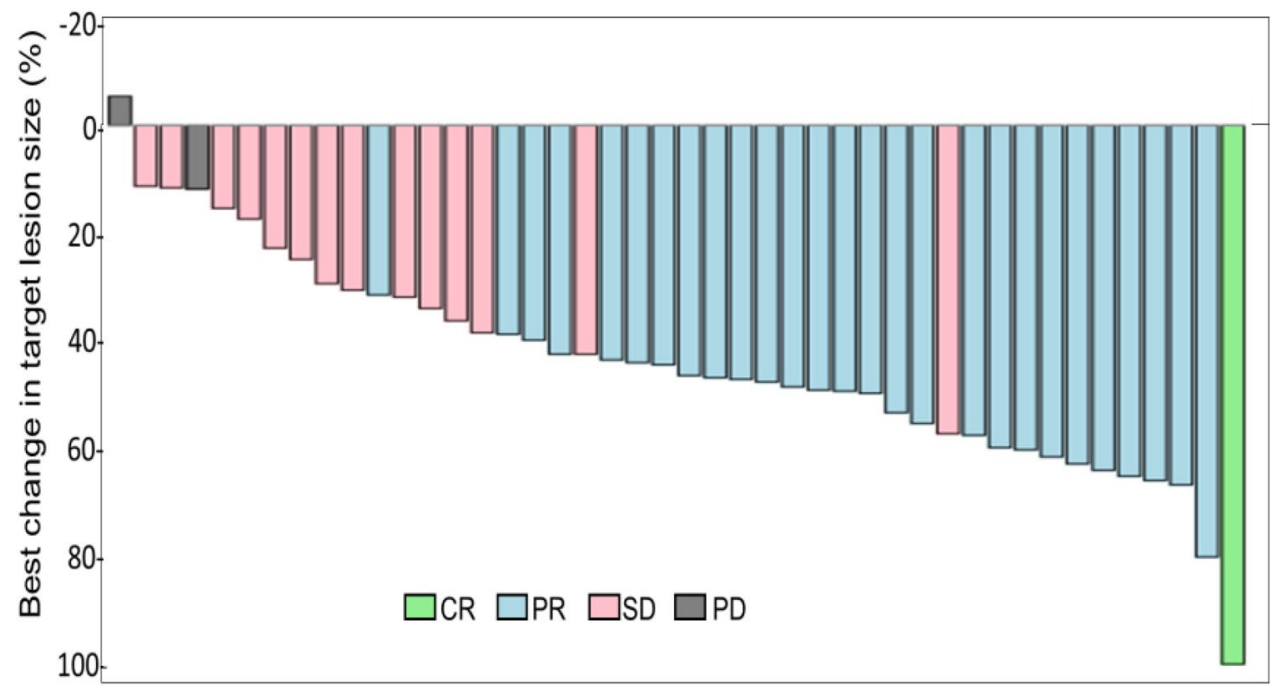

was $54.5 \%$. ETS was $70.5 \%$ (95\% CI 54.8-83.2). A waterfall plot of maximum tumor reduction rate is presented in Fig. 1. Median DoR was 45.4\% (95\% CI 37.8-49.9).

The median follow-up period was 25.4 months (ranging from 6 to 47 months). Median TTF was 8.1 months (95\% CI 5.3-10.1) based on 38 events (86.4\%) among 44 patients (Fig. 2a). Median PFS was 15.5 months (95\% CI 11.5-23.4) based on 28 events (63.6\%) among 44 patients (Fig. 2b). Median OS was 34.4 months (95\% CI 26.4 not reached) based on 19 deaths among 44 patients (43.2\%) (Fig. 2c).

\section{Subgroup analyses by primary tumor sidedness and RAS mutational status}

We evaluated the activity of FOLFOXIRI plus bevacizumab in an ad hoc analysis on the basis of primary tumor sidedness or $R A S$ status (Table 3). Following classification of the 44 patients in the FAS on the basis of primary tumor sidedness and $R A S$ mutation status, left-sided colon tumors were associated with a higher frequency of mutated $R A S$ tumors than right-sided colon tumors (47.7 vs. 6.8\%) (Supplementary Table 3 ). In stratification by sidedness, the ORR was numerically higher in right-sided colon tumors compared with left-sided ones (70.0 vs. $61.8 \%$, respectively). As for ETS and DoR, there was no difference between the two sides.

In stratification by $R A S$ status, the ORR was numerically higher in $R A S$ wild-type tumors compared with RAS mutated ones (73.3 vs. 62.5\%). ETS and median DoR were 73.3 and $47.6 \%$ in $R A S$ wild-type tumors and 70.8 and $45.2 \%$ in $R A S$ mutated tumors, respectively.

When analyzed on the basis of primary tumor sidedness, median PFS was 24.1 months (95\% CI 11.5-26.2) and 14.9 months (95\% CI 10.1-22.9) in right- and left-sided colon tumor cases, respectively, and the two survival curves intersected in the follow-up period (Supplementary Fig. 2a). Median OS was 26.4 months (95\% CI 26.2 not reached) and 34.4 months (95\% CI 23.6 not reached) in right- and left-sided colon tumor cases, respectively (Supplementary Fig. 2c). When analyzed according to $R A S$ mutational status, median PFS was 13.2 months (95\% CI 11.4-24.1) and 17.9 months (95\% CI 13.7-26.2) in $R A S$ wild-type and mutated tumor cases, respectively (Supplementary Fig. 2b), and the two survival curves crossed. Median OS was 37.1 months (95\% CI 23.6 not reached) and 29.8 months (95\% CI 26.2 not reached) in $R A S$ wild-type and mutated tumor cases, respectively (Supplementary Fig. 2d).

\section{Conversion surgery}

Fourteen patients (31.8\%) had conversion surgery performed with curative intent. Nine of these patients underwent surgery during the protocol therapy, and two patients did after the end of protocol therapy. The remaining three patients did during sequential treatment following the completion of protocol therapy. The median time to surgery from the initial day of protocol therapy was 182.5 days (range $87-316$ days).

$\mathrm{R} 0$ resection was performed in $10(22.7 \%)$ patients. Five of these patients were treated with 12 cycles of protocol therapy (two patients) or sequential treatment (three patients). When we examined the presence of ETS in ten patients with R0 resection, eight patients had ETS and two patients had no ETS. The surgical procedures (number of patients) performed on the four patients who could not achieve R0 resection included primary resection (3) and liver and lung resection (1). 
Fig. 2 Kaplan-Meier estimates of time to treatment failure (a), progression-free survival (b), and overall survival (c)

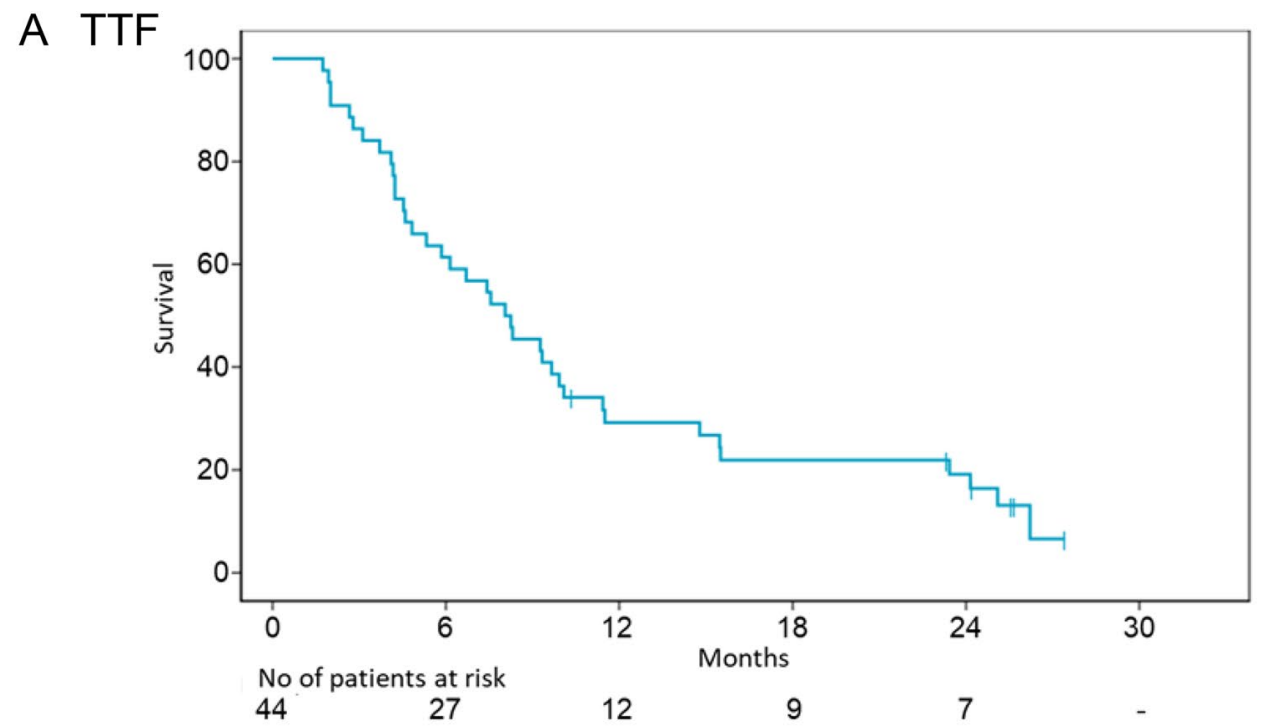

B PFS

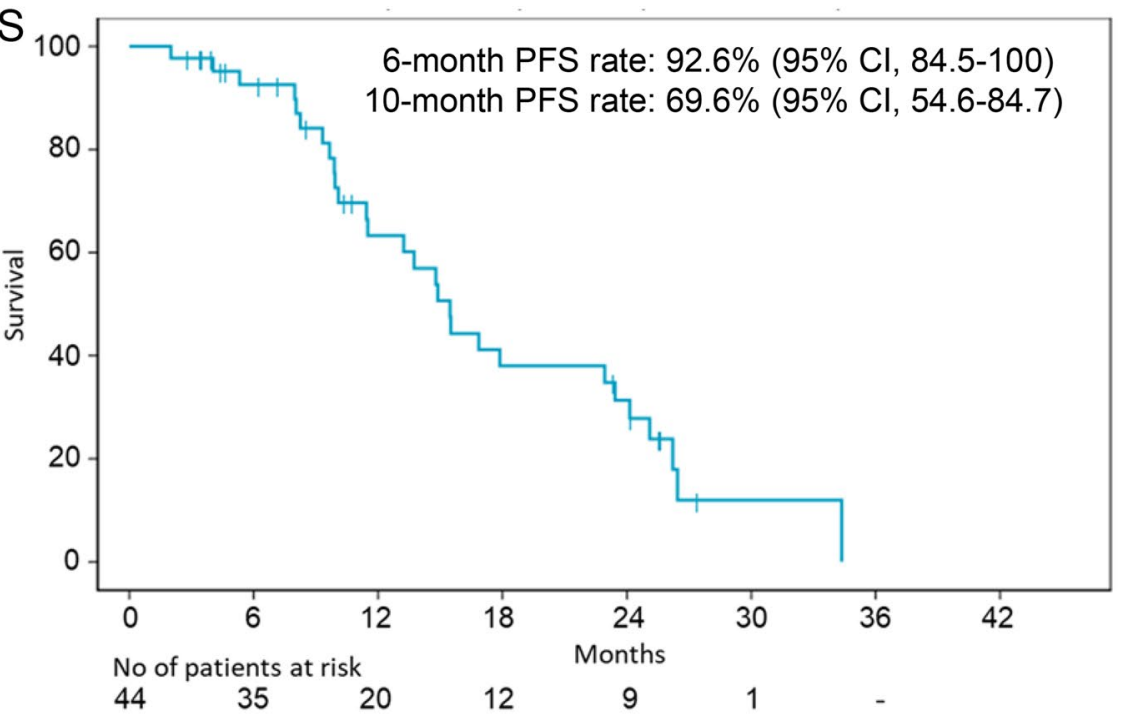

C OS

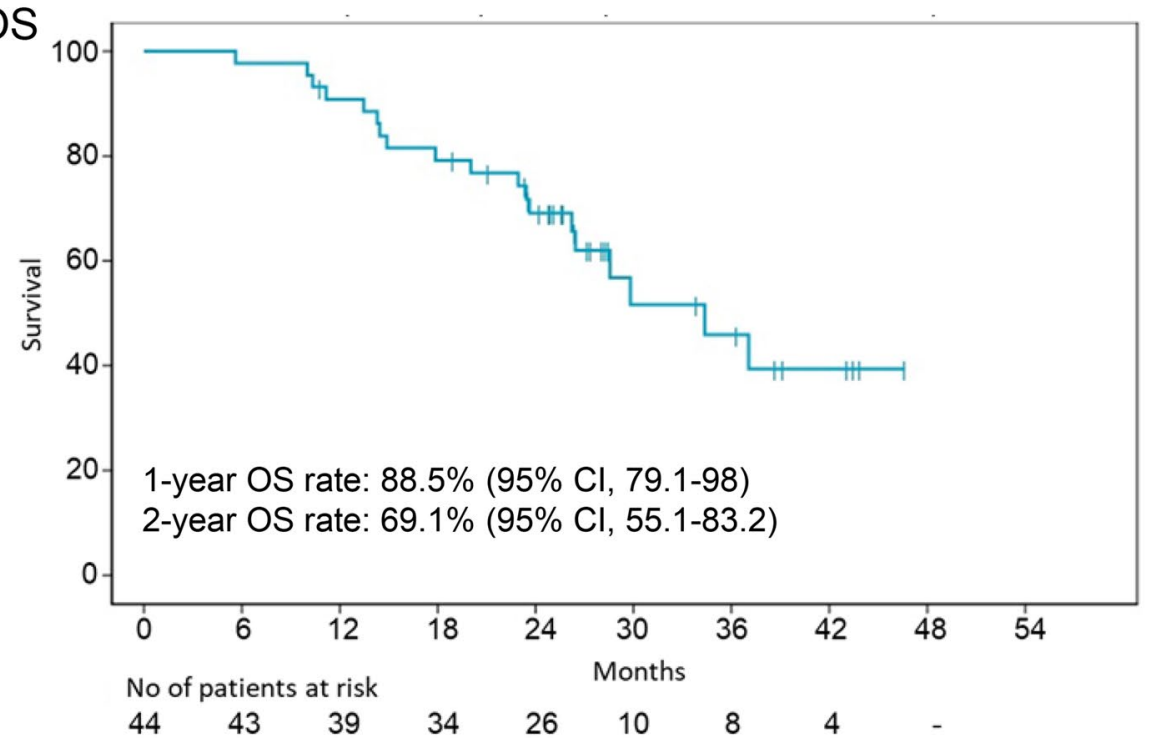


Table 4 Adverse events ( $\geq 5 \%)$ occurring in the safety sets $(n=46)$

\begin{tabular}{|c|c|c|}
\hline Adverse event & $\begin{array}{l}\text { All grade } \\
n(\%)\end{array}$ & $\begin{array}{l}\geq \mathrm{G} 3 \\
n(\%)\end{array}$ \\
\hline Anemia & $45(97.8)$ & $2(4.3)$ \\
\hline Neutropenia & $42(91.3)$ & $30(65.2)$ \\
\hline Leukopenia & $35(76.1)$ & $11(23.9)$ \\
\hline Thrombocytopenia & $26(56.5)$ & 0 \\
\hline Peripheral sensory neuropathy & $39(84.8)$ & $1(2.2)$ \\
\hline Malaise & $38(82.6)$ & 0 \\
\hline Alopecia & $36(78.3)$ & 0 \\
\hline Nausea & $35(76.1)$ & $5(10.9)$ \\
\hline Anorexia & $35(76.1)$ & $5(10.9)$ \\
\hline Diarrhea & $27(58.7)$ & $5(10.9)$ \\
\hline Fatigue & $27(58.7)$ & $2(4.3)$ \\
\hline Mucositis oral & $25(54.3)$ & $2(4.3)$ \\
\hline Hypertension & $25(54.3)$ & $4(8.7)$ \\
\hline Vomiting & $14(30.4)$ & $2(4.3)$ \\
\hline Febrile neutropenia & $12(26.1)$ & $12(26.1)$ \\
\hline Constipation & $9(19.6)$ & 0 \\
\hline Fever & $9(19.6)$ & $2(4.3)$ \\
\hline Hiccups & $8(17.4)$ & 0 \\
\hline Epistaxis & $7(15.2)$ & 0 \\
\hline $\begin{array}{l}\text { Palmar-plantar erythrodysesthesia } \\
\text { syndrome }\end{array}$ & $7(15.2)$ & 0 \\
\hline Skin hyperpigmentation & $5(10.9)$ & 0 \\
\hline Thromboembolic event & $5(10.9)$ & $2(4.3)$ \\
\hline Dry skin & $4(8.7)$ & 0 \\
\hline Hypoalbuminemia & $3(6.5)$ & 0 \\
\hline Abdominal pain & $3(6.5)$ & 0 \\
\hline Dysgeusia & $3(6.5)$ & 0 \\
\hline
\end{tabular}

\section{Toxicity}

All observed adverse events in the SAS are summarized in Table 4. Treatment-related grade 3 or 4 adverse events occurring in at least $10 \%$ of patients were as follows: neutropenia (65.2\%); febrile neutropenia (26.1\%); leukopenia (23.9\%); nausea (10.9\%); anorexia (10.9\%); and diarrhea (10.9\%). Two (16.7\%) of the 12 patients who developed febrile neutropenia were treated with G-CSF. No new adverse events were observed compared with previous historical studies.

In total, 14 serious adverse events occurred in 11 patients (23.9\%). Five of these adverse events were independent of protocol therapy. The remaining adverse events included infections (4), a thromboembolic event (1), an anastomosis fistula (1), ileus (1), nausea (1), and fever (1). All patients recovered from the serious adverse events at the end of the study. No treatment-related deaths occurred in the present study.

\section{Discussion}

Our BeTRI study confirmed the efficacy and safety of GONO-FOLFOXIRI plus bevacizumab in Japanese mCRC patients harboring $U G T 1 A 1$ wild $(* 1 / * 1)$ or single-heterozygous $(* 1 / * 6$ or $* 1 / * 28)$ genotypes.

Right-sided colon tumors are associated with a poorer prognosis compared with left-sided tumors $[16,17]$. Considering only patients with previously untreated mCRC receiving first-line doublet chemotherapy plus or minus bevacizumab, Loupakis et al. observed that ORR and PFS were statistically significantly higher in patients with leftsided tumors [16]. Interestingly, our ad hoc analysis showed that FOLFOXIRI plus bevacizumab treatment produced a numerically higher ORR, TTF, and PFS in right-sided colon tumor cases (compared with left-sided tumors). In rightsided colon tumor cases, ORR was the same, irrespective of $R A S$ mutational status. This finding supports the post hoc analysis of the TRIBE trial which found that FOLFOXIRI plus bevacizumab may be able to efficiently counteract the intrinsic aggressiveness of right-sided colon tumors [18].

FOLFOXIRI plus bevacizumab with an expected ORR of around $70 \%$ is an attractive strategy for conversion chemotherapy. Interestingly, conversion surgery $(31.8 \%)$ and R0 resection $(22.7 \%)$ rate in the present study were consistent with previous studies [9, 10, 14], despite the decreased dose intensity. The median time to surgery from the initial day of protocol therapy was 182.5 days (range $87-316$ days). Of the 14 patients who underwent surgery, the chemotherapy regimen immediately prior to surgery was FOLFOXIRI $(n=11)$, FOLFIRI $(n=1)$, and 5-fluorouracil and leucovorin $(n=2)$. Among the 10 patients who had undergone R0 resection, $50 \%$ of patients were treated with 12 or more cycles of protocol therapy. The ten patients who underwent $\mathrm{R} 0$ resection included two patients without ETS. This finding supports previously published meta-regression analysis data suggesting that a high number of chemotherapy cycles (close to 12) is associated with conversion surgery [19]. Therefore, a sufficient treatment time period may be required for $\mathrm{R} 0$ resection in unresectable or recurrent CRC.

Considering the safety profile of this drug regimen, $26.1 \%$ of Japanese patients developed febrile neutropenia in our BeTRI study, which was consistent with the results of the QUATTRO study. It should be noted that only two $(16.7 \%)$ of 12 patients were treated with G-CSF as a primary treatment or secondary prophylaxis of febrile neutropenia. The second cycle of treatment was performed without modification of dose or delay in $12(26 \%)$ patients. The median relative dose intensities of 5-fluorouracil, irinotecan, oxaliplatin, and bevacizumab were $72,69,62$, and $71 \%$, respectively. Compared with earlier studies $[9,14]$, the frequency of G-CSF use was extremely low. 
This may be related to the lower dose intensities in comparison with previous studies. The observed incidence of grade 3 or more diarrhea was low (10.9\%) compared with the two GONO-FOLFOXIRI plus bevacizumab studies (14-18.8\%) [4, 14], and was comparable with the QUATTRO study [9]. The major differences from the TRIBE trial was that target patients who harbored $U G T 1 A 1 * 1 / * 1$, $* 1 / * 6$, or $* 1 / * 28$ were included in our BeTRI and QUATTRO studies. This inclusion criterion may be the reason for the lower frequency of diarrhea. Our results indicate that the GONO-FOLFOXIRI regimen could be managed with appropriate dose reductions and treatment delays in Japanese patients harboring $U G T 1 A 1$ wild $(* 1 / * 1)$, or single-hetero $(* 1 / * 6$ or $* 1 / * 28)$ genotypes.

In clinical trials, chemotherapy is historically administered until disease progression, unacceptable toxicities, or patients' refusal. Oxaliplatin often causes cumulative neurotoxicity before clinical progression [20]. NCCN and ASCO guidelines recommend discontinuation of oxaliplatin from FOLFOX or CAPEOX three-four months after initiation of treatment or sooner for unacceptable neurotoxicity, with other drugs in the regimen maintained until time of tumor progression [6, 7]. In contrast, continuation of FOLFIRI induction treatment is recommended for at least as long as tumor shrinkage continues, or disease stabilization is maintained with tolerable toxicities [5]. In the TRIBE trial, 76\% of patients received secondary treatment, of which $63 \%$ were on irinotecan-containing regimens [4]. In our BeTRI study, a sequential treatment following FOLFOXIRI plus bevacizumab was not intentionally defined as maintenance treatment with 5-fluorouracil and leucovorin plus bevacizumab. A total of 27 (59\%) patients completed twelve cycles of protocol therapy. In fact, at the twelfth cycle, 18 (39.1\%) patients received FOLFOXIRI plus bevacizumab, six (13.0\%) patients received FOLFIRI plus bevacizumab, two (4.3\%) patients received FOLFIRI, and one (2.2\%) patient received infusional 5-fluorouracil and leucovorin plus bevacizumab. It should be noted that a FOLFIRI regimen was opted for eight (30.8\%) patients. Moreover, R0 resection was performed in $10(22.7 \%)$ patients including five patients treated with more than 12 cycles. These may have impacted on the encouraging lengths of 15.5 months for PFS and 37.1 months for OS. Taking the above into account, FOLFIRI appears to be suitable as a sequential treatment after three to four months of FOLFOXIRI or sooner for unacceptable neurotoxicity.

The current study had several potential limitations. A major weakness was that our trial was a single-arm, small sample size study with no comparators. Second, data on $B R A F$ mutational status were not available. Finally, the follow-up time was short for evaluation of OS. In the future, appropriate sequential treatment strategies following FOLFOXIRI induction treatment should be considered.
In conclusion, our BeTRI study demonstrated that GONO-FOLFOXIRI plus bevacizumab were highly beneficial and manageable with toxicities in selected Japanese patients with good performance status who can tolerate intensive treatment.

Acknowledgments We thank the patients who participated in this study and their families, the physicians and the medical staffs of the 16 sites registered in this study, and Dr. Hisayuki Endo, Dr. Yasuo Iwamoto, Dr. Takao Hinoi, and Dr. Satoshi Teramukai for comprising the independent data monitoring committee. We also thank Dr. Kunihisa Miyakawa and Dr. Masaki Matsusako for the central review. We acknowledge and appreciate the data analyses work carried out by Ms. Yoko Nakagawa and Mr. Hiromi Tsukada. We also appreciate the work of Mr. Tasuku Inaji in his duty as study manager in the Translational Research Center for Medical Innovation (TRI).

Author contributions All authors read and approved the final manuscript, and author contributions are in line with ICMJE guidelines. Substantial contributions: Planning and designing the study: KS, TN, TK. Acquisition of data: KS, TY, JN, TM, YY, TS, HN, IM, TF, MM, RY, KN, HT, TN, YK, MK, KT, MI, TK, TN. Writing the manuscript: KS, TY, TK, TN.

Funding This study was funded by Yakult Honsha Co., Ltd under contract. Yakult Honsha Co., Ltd played no role in the design, collection, analysis, or interpretation of the data, or writing of this manuscript.

\section{Compliance with ethical standards}

Conflict of interest KS has received speaker's honoraria from Chugai Pharmaceutical Co., Ltd., outside the submitted work; MK has received research funding from Chugai Pharmaceutical Co., Ltd., Yakult Honsha Co., Ltd., outside the submitted work. TN has received honoraria from Taiho Pharmaceutical Co., Ltd., and research funding from Taiho Pharmaceutical Co., Ltd., Chugai Pharmaceutical Co., Ltd., Ono Pharmaceutical Co., Ltd., Bristol Myers Squibb K.K., Eli Lilly Japan K.K., Daiichi Sankyo Co., Ltd., MSD K.K., Sumitomo Dainippon pharma Co., Ltd., outside the submitted work. TY, JN, TM, YY, TS, HN, IM, TF, MM, RY, KN, HT, TN, YK, KT, MI and TK declares no conflicts of interest.

Ethical approval This study was conducted by the Translational Research Center for Medical Innovation (TRI) in accordance with the Declaration of Helsinki and Clinical Trials Act in Japan. The study protocol was approved by the Institutional Review Board of each participating institution and Certified Review Board. All patients provided written informed consent. This study was registered with UMIN Clinical Trial Registry (Trial Identifier UMIN000017102), the Japan Registry of Clinical Trials (jRCTs061180021), and ClinicalTrials.gov (NCT02497157).

Informed consent Informed consent was obtained from all individual participants included in this study.

Availability of data and material The datasets generated during the current study are not publicly available because the informed consent form signed by the participants did not address an individual data sharing statement.

Open Access This article is licensed under a Creative Commons Attribution 4.0 International License, which permits use, sharing, 
adaptation, distribution and reproduction in any medium or format, as long as you give appropriate credit to the original author(s) and the source, provide a link to the Creative Commons licence, and indicate if changes were made. The images or other third party material in this article are included in the article's Creative Commons licence, unless indicated otherwise in a credit line to the material. If material is not included in the article's Creative Commons licence and your intended use is not permitted by statutory regulation or exceeds the permitted use, you will need to obtain permission directly from the copyright holder. To view a copy of this licence, visit http://creativecommons .org/licenses/by/4.0/.

\section{References}

1. Bray F, Ferlay J, Soerjomataram I et al (2018) Global cancer statistics 2018: GLOBOCAN estimates of incidence and mortality worldwide for 36 cancers in 185 countries. CA Cancer J Clin 68(6):394-424. https://doi.org/10.3322/caac.21492

2. House MG, Ito H, Gonen M et al (2010) Survival after hepatic resection for metastatic colorectal cancer: trends in outcomes for 1600 patients during two decades at a single institution. J Am Coll Surgeons 210(5):744-752. https://doi.org/10.1016/j.jamco 1lsurg.2009.12.040 (752-745)

3. Loupakis F, Cremolini C, Masi G et al (2014) Initial therapy with FOLFOXIRI and bevacizumab for metastatic colorectal cancer. $\mathrm{N}$ Engl J Med 371(17):1609-1618. https://doi.org/10.1056/NEJMo a1403108

4. Cremolini C, Loupakis F, Antoniotti C et al (2015a) FOLFOXIRI plus bevacizumab versus FOLFIRI plus bevacizumab as first-line treatment of patients with metastatic colorectal cancer: updated overall survival and molecular subgroup analyses of the openlabel, phase 3 TRIBE study. Lancet Oncol 16(13):1306-1315. https://doi.org/10.1016/s1470-2045(15)00122-9

5. Van Cutsem E, Cervantes A, Adam R et al (2016) ESMO consensus guidelines for the management of patients with metastatic colorectal cancer. Ann Oncol 27(8):1386-1422. https://doi. org/10.1093/annonc/mdw235

6. National Comprehensive Cancer Network. NCCN clinical practice guidelines in oncology-colon cancer, version 4.2020. Accessed 17 July 2020

7. Chiorean EG, Nandakumar G, Fadelu T et al (2020) Treatment of patients with late-stage colorectal cancer: ASCO ResourceStratified Guideline. JCO Global Oncol 6:414-438. https://doi. org/10.1200/jgo.19.00367

8. Hashiguchi Y, Muro K, Saito Y et al (2020) Japanese Society for Cancer of the Colon and Rectum (JSCCR) guidelines 2019 for the treatment of colorectal cancer. Int J Clin Oncol 25(1):1-42. https ://doi.org/10.1007/s10147-019-01485-z

9. Oki E, Kato T, Bando $\mathrm{H}$ et al (2018) A multicenter clinical phase II study of FOLFOXIRI plus Bevacizumab as first-line therapy in patients with metastatic colorectal cancer: QUATTRO Study. Clin Colorectal Cancer 17(2):147-155. https://doi.org/10.1016/j. clcc.2018.01.011

10. Satake H, Sunakawa Y, Miyamoto Y et al (2018) A phase II trial of 1st-line modified-FOLFOXIRI plus bevacizumab treatment for metastatic colorectal cancer harboring RAS mutation: JACCRO CC-11. Oncotarget 9(27):18811-18820. https://doi.org/10.18632 /oncotarget.24702

11. Shui L, Wu YS, Lin $\mathrm{H}$ et al (2018) Triplet Chemotherapy (FOLFOXIRI) plus Bevacizumab versus doublet chemotherapy (FOLFOX/FOLFIRI) plus Bevacizumab in conversion therapy for metastatic colorectal cancer: a meta-analysis. Cell Physiol Biochem 48(5):1870-1881. https://doi.org/10.1159/000492508

12. Ando Y, Saka H, Ando M et al (2000) Polymorphisms of UDPglucuronosyltransferase gene and irinotecan toxicity: a pharmacogenetic analysis. Cancer Res 60(24):6921-6926

13. Minami H, Sai K, Saeki M et al (2007) Irinotecan pharmacokinetics/pharmacodynamics and UGT1A genetic polymorphisms in Japanese: roles of UGT1A1* 6 and $* 28$. Pharmacogenet Genomics 17(7):497-504. https://doi.org/10.1097/FPC.0b013e328014341f

14. Masi G, Loupakis F, Salvatore L et al (2010) Bevacizumab with FOLFOXIRI (irinotecan, oxaliplatin, fluorouracil, and folinate) as first-line treatment for metastatic colorectal cancer: a phase 2 trial. Lancet Oncol 11(9):845-852. https://doi.org/10.1016/s1470 -2045(10)70175-3

15. Cassidy J, Clarke S, Diaz-Rubio E et al (2008) Randomized phase III study of capecitabine plus oxaliplatin compared with fluorouracil/folinic acid plus oxaliplatin as first-line therapy for metastatic colorectal cancer. J Clin Oncol 26(12):2006-2012. https://doi. org/10.1200/jco.2007.14.9898

16. Loupakis F, Yang D, Yau L et al (2015) Primary tumor location as a prognostic factor in metastatic colorectal cancer. J Natl Cancer I. https://doi.org/10.1093/jnci/dju427

17. Petrelli F, Tomasello G, Borgonovo K et al (2017) Prognostic survival associated with left-sided vs right-sided colon cancer: A systematic review and meta-analysis. JAMA Oncol 3(2):211-219. https://doi.org/10.1001/jamaoncol.2016.4227

18. Cremolini C, Loupakis F, Antoniotti C et al (2015b) Early tumor shrinkage and depth of response predict long-term outcome in metastatic colorectal cancer patients treated with first-line chemotherapy plus bevacizumab: results from phase III TRIBE trial by the GruppoOncologico del Nord Ovest. Ann Oncol 26(6):11881194. https://doi.org/10.1093/annonc/mdv112

19. Tomasello G, Petrelli F, Ghidini M et al (2017) FOLFOXIRI plus Bevacizumab as conversion therapy for patients with initially unresectable metastatic colorectal cancer: A systematic review and pooled analysis. JAMA Oncol 3(7):e170278. https://doi. org/10.1001/jamaoncol.2017.0278

20. Kidwell KM, Yothers G, Ganz PA et al (2012) Long-term neurotoxicity effects of oxaliplatin added to fluorouracil and leucovorin as adjuvant therapy for colon cancer: results from National Surgical Adjuvant Breast and Bowel Project trials C-07 and LTS-01. Cancer 118(22):5614-5622. https://doi.org/10.1002/cncr.27593

Publisher's Note Springer Nature remains neutral with regard to jurisdictional claims in published maps and institutional affiliations. 


\section{Affiliations}

\section{Katsunori Shinozaki ${ }^{1}$ (D) Takeshi Yamada ${ }^{2}$. Junichiro Nasu ${ }^{3} \cdot$ Toshihiko Matsumoto $^{4}$. Yasuhiro Yuasa ${ }^{5}$ Takeshi Shiraishi $^{6}$. Hiroaki Nagano ${ }^{7}$. Ichiro Moriyama ${ }^{8} \cdot$ Toshiyoshi Fujiwara $^{9}$. Masashi Miguchi ${ }^{10}$. Ryosuke Yoshida $^{11} \cdot$ Kimiyasu Nozaka $^{12} \cdot$ Hiroaki Tanioka $^{13} \cdot$ Takeshi Nagasaka $^{13} \cdot$ Yasuro Kurisu $^{14}$. Michiya Kobayashi ${ }^{15} \cdot K_{\text {Kenji Tsuchihashi }}{ }^{16} \cdot$ Michio Inukai $^{3} \cdot$ Takashi Kikuchi $^{17} \cdot$ Tomohiro Nishina $^{18}$}

1 Division of Clinical Oncology, Hiroshima Prefectural Hospital, 1-5-54 Ujinakanda, Hiroshima 734-8530, Japan

2 Division of Gastroenterology, University of Tsukuba, Tsukuba 305-8577, Japan

3 Department of Internal Medicine, Okayama Saiseikai General Hospital, Okayama 700-8501, Japan

4 Department of Internal Medicine, Himeji Red Cross Hospital, Himeji 670-8540, Japan

5 Department of Surgery, Tokushima Red Cross Hospital, Tokushima 773-8502, Japan

6 Department of Medical Oncology, Matsuyama Red Cross Hospital, Matsuyama 790-8520, Japan

7 Department of Gastroenterological Surgery, Yamaguchi University Graduate School of Medicine, Ube 755-8505, Japan

8 Innovative Cancer Center, Shimane University Hospital, Izumo 693-0021, Japan

9 Department of Gastroenterological Surgery, Okayama University Graduate School of Medicine, Dentistry and Pharmaceutical Sciences, Okayama 700-8530, Japan
10 Department of Surgery, Hiroshima City Asa Citizens Hospital, Hiroshima 731-0293, Japan

11 Department of Surgery, Okayama Rosai Hospital, Okayama 702-8055, Japan

12 Department of Surgery, Sanin Rosai Hospital, Yonago 683-8605, Japan

13 Department of Clinical Oncology, Kawasaki Medical School Hospital, Kurashiki 701-0192, Japan

14 Department of Surgery, Hamada Medical Center, Hamada 697-8511, Japan

15 Cancer Treatment Center, Kochi Medical School Hospital, Nankoku 783-8505, Japan

16 Department of Hematology, Oncology and Cardiovascular Medicine, Kyushu University Hospital, Fukuoka 812-8582, Japan

17 Foundation for Biomedical Research and Innovation at Kobe for Medical Innovation, Kobe 650-0047, Japan

18 Department of Gastrointestinal Medical Oncology, National Hospital Organization Shikoku Cancer Center, Matsuyama 791-0280, Japan 